\title{
Treatment Patterns and Mortality Risk among Elderly Patients with Metastatic Triple Negative Breast Cancer in the United States: An Observational Cohort Study Using SEER-Medicare Data
}

\author{
Sacha Satram-Hoang1 ${ }^{*}$, Preeti Bajaj ${ }^{2}$, Alisha Stein'2, Patricia Cortazar ${ }^{2}$, Faiyaz Momin'1, \\ Carolina Reyes ${ }^{2}$ \\ ${ }^{1}$ Q.D. Research, Inc., Granite Bay, CA, USA \\ ${ }^{2}$ Genentech, Inc., South San Francisco, CA, USA \\ Email: *sacha@qdresearch.com
}

How to cite this paper: Satram-Hoang, S., Bajaj, P., Stein, A., Cortazar, P., Momin, F. and Reyes, C. (2019) Treatment Patterns and Mortality Risk among Elderly Patients with Metastatic Triple Negative Breast Cancer in the United States: An Observational Cohort Study Using SEER-Medicare Data. Journal of Cancer Therapy, 10, 117-133. https://doi.org/10.4236/jct.2019.102009

Received: January 15, 2019

Accepted: February 12, 2019

Published: February 15, 2019

Copyright $\odot 2019$ by author(s) and Scientific Research Publishing Inc. This work is licensed under the Creative Commons Attribution International License (CC BY 4.0).

http://creativecommons.org/licenses/by/4.0/

\begin{abstract}
Purpose: Triple negative breast cancer is more aggressive than other breast cancer subtypes and accounts for up to $20 \%$ of all breast cancers. Despite the poorer prognosis, there are no approved targeted treatments available and chemotherapy remains the only choice. We examined treatment patterns and outcomes among elderly metastatic triple-negative breast cancer (mTNBC) patients in routine clinical practice. Methods. Patients were identified from the linked SEER-Medicare database between 1/1/2001 and 12/31/2013 and included de novo Stage IV $(\mathrm{n}=776)$ and patients with distant metastasis followed an initial diagnosis of Stage I - III disease $(n=1851)$. Kaplan-Meier analyses and time-varying Cox proportional hazards regression were used to assess overall survival (OS). Results. The mean age at metastatic diagnosis was 77.6 years and 1259 (48\%) patients received chemotherapy. Compared to $<70$ year olds, $\geq 70$ year olds had worse performance status, higher comorbidity burden, and were less likely to receive chemotherapy ( $45 \%$ vs. $66 \%$ ). Patients treated with chemotherapy had increased OS compared to untreated patients, and the survival advantage was more pronounced in the $<70$ year olds with a 6-month longer unadjusted OS compared to the $\geq 70$ cohort (log rank $\mathrm{p}<$ $0.0001)$. This finding was supported in the adjusted multivariate model which showed a $46 \%$ increased risk of death for untreated patients in the $<70$ year olds and a $17 \%$ increased risk of death for untreated patients in the $\geq 70$ year olds (vs. treated). Conclusions. In this real-world analysis, $48 \%$ of elderly mTNBC patients did not receive chemotherapy and a greater proportion were
\end{abstract}


untreated in the $\geq 70$ year old cohort (55\%). Although the survival benefits of chemotherapy were greater in the younger cohort, the benefits of treatment persisted in $\geq 70$ year olds. These findings suggest opportunities exist to improve the clinical treatment of elderly mTNBC patients.

\section{Keywords}

Triple Negative Breast Cancer, Elderly Patients, Chemotherapy, Survival

\section{Introduction}

Breast cancer is the most common type of cancers affecting women in the United States, accounting for approximately 252,710 new cases in 2017 [1]. The risk increases with age, and about one-third of female breast cancers are diagnosed in patients older than 70 years of age [2]. Approximately $6 \%$ of women will present with metastatic disease at diagnosis (de novo Stage IV disease) and roughly $30 \%$ of women diagnosed with early stage disease will experience a distant recurrence [3]. The majority of deaths from breast cancer result from recurrent or metastatic disease. In 2017, there will be an estimated 40,610 deaths, making breast cancer the second leading cause of deaths from cancers among women [1].

The triple-negative breast cancer (TNBC) subtype accounts for $12 \%-17 \%$ of all breast cancers and is characterized by the lack of expression of estrogen receptor (ER), progesterone receptor (PR) and human epidermal growth factor receptor 2 (HER2) [4]. Although less than $6 \%$ of breast cancers are associated with breast cancer gene (BRCA) mutations, around $20 \%$ of TNBC patients have a BRCA mutation [5]. TNBC is typically diagnosed at an earlier age, is more prevalent among Hispanic and African women, and is more aggressive with a high risk of metastasis and deaths within 5 years after diagnosis [4] [6] [7]. The triple-negative phenotype is unresponsive to endocrine therapy, and until recently, there have been no targeted therapies approved by the FDA, making chemotherapy the only treatment option.

The therapeutic goal at this advanced stage of disease is palliative: to prolong survival and improve quality of life. The National Comprehensive Cancer Network (NCCN) guidelines for breast cancer have no age limit recommendation for chemotherapy but state that there are limited data to make chemotherapy recommendations for patients older than 70 years and that treatment should be individualized with consideration of comorbid conditions [8]. There are limited data among patients older than 70 years as chemotherapy clinical trials usually include younger and healthier cancer patients, and fewer than $4 \%$ of clinical trial participants are older than 70 [9] [10]. Age has been shown to be a barrier to receiving chemotherapy due to physician and patient concerns about treatment toxicity [11] [12] [13]. Elderly patients have poorer outcomes compared to younger patients, and this may be related to "under-treatment" in the elderly population [2] [14]. 
Few population-based studies of treatment patterns and survival outcomes among metastatic TNBC patients exist. It is important to understand whether the benefits of chemotherapy are maintained in elderly mTNBC patients in the real-world setting. The objective of this study was to assess chemotherapy treatment patterns and outcomes to better understand the age-related differences among TNBC patients and the unmet need in a real-world setting.

\section{Materials and Methods}

\subsection{Data Source}

The linked Surveillance, Epidemiology, and End Results (SEER)-Medicare database combines cancer registry data with administrative Medicare files from the Centers for Medicare and Medicaid Services. It contains more than 3.3 million persons with cancer. SEER is a nationally representative collection of population-based cancer registries from diverse geographic areas covering approximately $26 \%$ of the United States population. The majority of persons aged 65 years and older in SEER are successfully matched to their Medicare enrollment files [15]. Details of the SEER-Medicare database have been published previously [15]. Briefly, the database combines clinical, demographic, cancer diagnosis, survival, and cause of death information with medical claims (hospital, physician, outpatient, home health, and hospice bills) for adults 65 years and older with cancer. All Medicare beneficiaries receive Part A coverage (inpatient care, skilled nursing, home healthcare and hospice care) and approximately $95 \%$ of beneficiaries subscribe to Part B (outpatient and physician services). The database linkage used in this study included cancer cases diagnosed until 2011 with Medicare claims through 2013. Institutional review board (IRB) approval was waived because the National Institutes of Health's Office of Human Subjects Research has determined that analyses using SEER-Medicare data are exempt from requiring further IRB review and approval.

\subsection{Study Population}

Patients with a first primary breast cancer were identified using the SEER variables indicating the cancer site and order of incident cancer diagnoses. Data on ER and PR status have been collected since 1990 and HER2 since 2010 in SEER. HER2 status was not available before the year 2010, so the presence of Medicare claims for HER2 targeted therapies, i.e., trastuzumab (Herceptin ${ }^{\oplus}$, Roche) and lapatinib (Tykerb ${ }^{\circ}$, Glaxo Smith Kline) were used as a proxy for HER2 positive status, and absence of these claims was assumed to indicate HER2 negative status. A case was defined as triple-negative if ER, PR, and HER2 were negative. Staging of primary breast cancer was based on the American Joint Committee on Cancer Criteria (AJCC), $6^{\text {th }}$ edition in SEER. Since SEER does not include information on disease progression, Medicare claims data were used to identify patients with distant metastasis following an initial diagnosis of Stage I - III breast cancer. The first-listed International Classification of Disease, $9^{\text {th }}$ Revi- 
sion, Clinical Modification (ICD-9-CM) code indicating a secondary cancer (197.XX-198.XX), excluding breast $(198.81,198.82)$ and lymph node (196.XX) [16] [17], were used to classify patients in the non-de novo metastatic group.

See Figure 1 for the schematic of the inclusion/exclusion process. Patients were diagnosed with a first primary metastatic triple-negative breast cancer between January 1, 2001 and December 31, 2011, $\geq 66$ years at the time of diagnosis, and enrolled in Medicare Parts A and B for a full 12 months prior to diagnosis date. Patients were excluded if breast cancer was diagnosed at the time of death or autopsy, and if enrolled in a health maintenance organization (HMO) any time within the 12 months prior to diagnosis as HMO claims are unavailable in the dataset. There were 776 de novo Stage IV patients and 1851 patients with non-de novo metastatic disease identified using the algorithm described above.

\subsection{Study Variables}

Key study measures include patient demographics (age, race/ethnicity, marital status, income, education level and geographic region), clinical characteristics (tumor characteristics, comorbidity burden, poor performance indicators), and treatment information. Performance status measures, such as Eastern Cooperative Oncology Group (ECOG) are not available in the dataset so Medicare claims were used to identify poor performance indicators (PPI) which include oxygen and related respiratory supplies, wheelchair and supplies, home health agency services, and skilled nursing facility services occurring in the 12 months prior to metastatic diagnosis date [18]. The National Cancer Institute (NCI) comorbidity index [19], the gold-standard in SEER-Medicare, was used to assess comorbidity burden using diagnosis and procedure codes in the Medicare claims files to identify the 15 non-cancer comorbidities from the Charlson Comorbidity Index [20] that occurred in the 12 months prior to metastatic diagnosis date.

In the Medicare claims files, ICD-9-CM procedure codes were used to identify chemotherapy administration while the Healthcare Common Procedural Coding System (HCPCS) "J" codes were used to identify the specific intravenous chemotherapy administered [21]. The first claim for chemotherapy was required to appear within six months of metastatic diagnosis date for a patient to be considered in the "treated" cohort. Patients with a history of breast cancer surgery (lumpectomy and mastectomy) and radiation therapy, were identified by searching Medicare claims for ICD-9 procedure codes and Current Procedural Terminology (CPT) procedure codes from initial diagnosis date of first primary breast cancer through the end of follow-up.

\subsection{Statistical Analysis}

Patient characteristics were compared by age and treatment status using the Chi-square test for categorical variables and ANOVA or t-test for continuous variables, with tests of significance measured at a p-value $<0.05$. The primary endpoint, overall survival (OS) was measured from date of metastatic diagnosis 


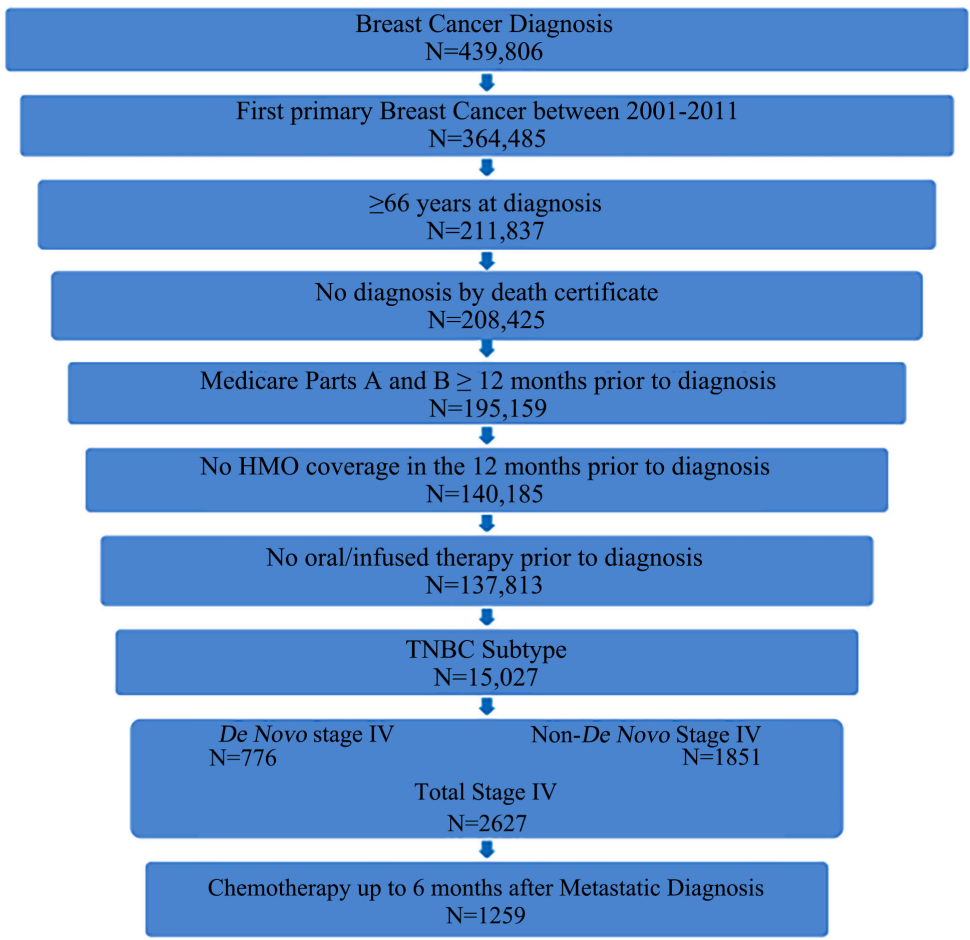

Figure 1. Cohort inclusion/exclusion criteria.

to date of death; if still alive, patients were censored at the end of the follow-up period (December 31, 2013) or until Medicare claims were no longer available. Unadjusted overall survival was assessed using Kaplan-Meier survival analysis. A time-varying Cox proportional hazards regression model with chemotherapy treatment as a time-dependent factor was used to account for variation in treatment initiation between patients and to minimize the introduction of immortal time bias into the analysis (period of follow-up time during which death cannot occur) [22]. In the time-varying Cox model, all patients belong to the "not treated" group and only switched to the "treated" group at the time of treatment receipt. This Cox model is used to explore predictors of overall risk of death adjusting for potential confounding variables which were selected based on a priori beliefs that these factors are associated with receipt of treatment. The fully adjusted model included age, race, marital status, education, income, geographic region, initial stage at diagnosis, year of diagnosis, poor performance, comorbidity score, radiotherapy, surgery, and chemotherapy. In these survival analyses, comparisons were made between the chemotherapy treated versus not treated patients, stratified by age $<70$ years and $\geq 70$ years. All statistical analyses were performed using SAS software, version 9.1.3 (SAS Institute Inc., Cary, North Carolina).

\section{Results}

\subsection{Patient Characteristics}

The demographic and clinical characteristics of the cohort are presented in $\mathrm{Ta}$ - 
ble 1 . The mean age at metastatic diagnosis was 77.6 years. The majority of metastatic patients were in the older cohort of $\geq 70$ year olds (86\%) compared to the younger cohort of $<70$ year olds (14\%). Patients in the older cohort were more likely to be white ( $78 \%$ vs. $71 \%$ ) while the younger cohort had a higher proportion of patients of African ancestry (25\% vs. $16 \%$ ). Patients in the older cohort were also more likely to be widowed ( $46 \%$ vs. $25 \%$ ), have a higher education level, and higher income level compared to their younger counterparts. In regards to clinical characteristics, patients in the younger cohort were more likely to be diagnosed with de novo Stage IV disease ( $42 \%$ vs. $28 \%$ ) while the older cohort contained more patients who experienced distant metastasis following an initial diagnosis of Stage I - III breast cancer (72\% vs. 58\%). Compared to the younger cohort, the patients in the older cohort had poorer performance and a higher comorbidity burden.

\subsection{Treatment Patterns}

There was no difference in radiotherapy treatment rates between age cohorts, however patients in the older cohort were more likely to have a history of breast cancer surgery compared to the younger cohort. Overall, about $48 \%$ of TNBC patients received treatment with chemotherapy within 6 months after metastatic diagnosis, and the proportion treated was higher in the younger cohort compared to the older cohort (66\% vs. $45 \%$ ). Cyclophosphamide (29\%), paclitaxel (23\%), docetaxel (23\%), doxorubicin (20\%) carboplatin (11\%) and capecitabine (10\%) -based regimens were the most common first-line chemotherapies administered in the metastatic setting (Table 2). Patients in the older cohort were more likely to receive capecitabine-based therapy; all other treatment regimens were more common in the younger cohort of patients.

\subsection{Survival}

The median unadjusted OS was 8.8 months (95\% CI: 8.0 - 9.8) for all metastatic TNBC patients (Figure 2). Patients who received treatment with chemotherapy had a higher unadjusted median OS (12.8 months, 95\% CI: 11.5 - 13.9) compared to untreated patients (4.9 months, $95 \%$ CI: 4.2 - 5.8). When stratifying by age, the survival advantage was more pronounced among treated patients in the younger cohort (17.7 months, $95 \%$ CI: 13.4 - 24.8 vs.) compared to treated patients within the older cohort (11.7 months, 95\% CI: 10.5 - 13.4 vs. 5.0 months, 95\% CI: 4.2 - 5.9).

The time-varying Cox model (Table 3 ) showed no statistically significant mortality risk difference for untreated vs. treated patients in the overall cohort of metastatic patients $(\mathrm{HR}=1.095 ; 95 \% \mathrm{CI}=1.00-1.20)$. However, when stratifying by age group, a $46 \%$ increased risk of death for untreated patients in the $<70$ year old cohort and a $17 \%$ increased risk of death for untreated patients in the $\geq 70$ year olds (vs. chemotherapy treated patients) were observed. Other factors in the model found to be predictive of increased mortality risk included: increasing initial stage at diagnosis, increasing comorbidity score, and presence of 
PPI. Prior radiotherapy and breast cancer surgery were associated with lower mortality risks.

Table 1. Demographic and clinical characteristics by age.

\begin{tabular}{|c|c|c|c|c|c|c|c|}
\hline & \multicolumn{2}{|c|}{$\begin{array}{l}\text { Total metastatic } \\
\qquad \mathrm{N}=2627\end{array}$} & \multicolumn{2}{|c|}{$\begin{array}{c}<70 \text { years } \\
N=359\end{array}$} & \multicolumn{2}{|c|}{$\begin{array}{l}\geq 70 \text { years } \\
N=2268\end{array}$} & \multirow[t]{2}{*}{$p$ value } \\
\hline & $\mathrm{n}$ & $\%$ & $\mathrm{n}$ & $\%$ & $\mathrm{n}$ & $\%$ & \\
\hline Mean age $(95 \% \mathrm{CI})$ & 77.57 & $77.3-77.8$ & 67.67 & $67.6-67.8$ & 79.14 & $78.9-79.4$ & $<0.0001$ \\
\hline \multicolumn{8}{|l|}{ Race/ethnicity } \\
\hline White & 2032 & 77.4 & 255 & 71.0 & 1777 & 78.4 & 0.0002 \\
\hline Black & 460 & 17.5 & 90 & 25.1 & 370 & 16.3 & \\
\hline Other/unknown & 135 & 5.1 & 14 & 3.9 & 121 & 5.3 & \\
\hline \multicolumn{8}{|l|}{ Marital status } \\
\hline Single & 219 & 8.3 & 48 & 13.4 & 171 & 7.5 & $<0.0001$ \\
\hline Married & 946 & 36.0 & 162 & 45.1 & 784 & 34.6 & \\
\hline Separated/divorced & 250 & 9.5 & 44 & 12.3 & 206 & 9.1 & \\
\hline Widowed & 1134 & 43.2 & 90 & 25.1 & 1044 & 46.0 & \\
\hline Unknown & 78 & 3.0 & 15 & 4.2 & 63 & 2.8 & \\
\hline \multicolumn{8}{|c|}{$\%$ of adults with some education } \\
\hline $0-50$ & 906 & 34.5 & 144 & 40.1 & 762 & 33.6 & 0.0077 \\
\hline $51-100$ & 1665 & 63.4 & 203 & 56.5 & 1462 & 64.5 & \\
\hline Unknown & 56 & 2.1 & 12 & 3.3 & 44 & 1.9 & \\
\hline \multicolumn{8}{|l|}{ Median income quartiles } \\
\hline 1-Low & 643 & 24.5 & 118 & 32.9 & 525 & 23.1 & 0.0002 \\
\hline 2 & 644 & 24.5 & 85 & 23.7 & 559 & 24.6 & \\
\hline 3 & 643 & 24.5 & 77 & 21.4 & 566 & 25.0 & \\
\hline 4-High & 641 & 24.4 & 67 & 18.7 & 574 & 25.3 & \\
\hline Unknown & 56 & 2.1 & 12 & 3.3 & 44 & 1.9 & \\
\hline \multicolumn{8}{|l|}{ Geographic region } \\
\hline Midwest & 347 & 13.2 & 54 & 15.0 & 293 & 12.9 & 0.0123 \\
\hline Northeast & 187 & 7.1 & 28 & 7.8 & 159 & 7.0 & \\
\hline South & 958 & 36.5 & 103 & 28.7 & 855 & 37.7 & \\
\hline West & 1135 & 43.2 & 174 & 48.5 & 961 & 42.4 & \\
\hline \multicolumn{8}{|l|}{ Stage at initial diagnosis } \\
\hline Stage I & 678 & 25.8 & 68 & 18.9 & 610 & 26.9 & $<0.0001$ \\
\hline Stage II & 785 & 29.9 & 91 & 25.3 & 694 & 30.6 & \\
\hline Stage III & 388 & 14.8 & 50 & 13.9 & 338 & 14.9 & \\
\hline Stage IV & 776 & 29.5 & 150 & 41.8 & 626 & 27.6 & \\
\hline \multicolumn{8}{|l|}{ PPI } \\
\hline No & 2288 & 87.1 & 324 & 90.3 & 1964 & 86.6 & 0.0550 \\
\hline
\end{tabular}




\section{Continued}

$\begin{array}{lllllll}\text { Yes } & 339 & 12.9 & 35 & 9.7 & 304 & 13.4\end{array}$

NCI comorbidity score

$\begin{array}{cccccccc}0 & 1483 & 56.5 & 227 & 63.2 & 1256 & 55.4 & 0.0116 \\ 1 & 643 & 24.5 & 72 & 20.1 & 571 & 25.2 & \\ 2 & 257 & 9.8 & 24 & 6.7 & 233 & 10.3 \\ \geq 3 & 244 & 9.3 & 36 & 10.0 & 208 & 9.2\end{array}$

Radiation therapy

$\begin{array}{llllllll}\text { No } & 1082 & 41.2 & 136 & 37.9 & 946 & 41.7 & 0.1709 \\ \text { Yes } & 1545 & 58.8 & 223 & 62.1 & 1322 & 58.3 & \end{array}$

Breast cancer surgery

$\begin{array}{lrrrrrrr}\text { No } & 544 & 20.7 & 108 & 30.1 & 436 & 19.2 & <0.0001 \\ \text { Yes } & 2083 & 79.3 & 251 & 69.9 & 1832 & 80.8 & \end{array}$

Chemotherapy

$\begin{array}{llllllll}\text { No } & 1368 & 52.1 & 123 & 34.3 & 1245 & 54.9 & <0.0001 \\ \text { Yes } & 1259 & 47.9 & 236 & 65.7 & 1023 & 45.1 & \end{array}$

Abbreviations: NCI: National Cancer Institute; PPI: poor performance indicators.

Table 2. First-line chemotherapy in metastatic setting.

\begin{tabular}{|c|c|c|c|c|c|c|}
\hline \multirow[t]{2}{*}{ Chemotherapy } & \multicolumn{2}{|c|}{$\begin{array}{l}\text { Total treated } \\
\mathrm{N}=1259\end{array}$} & \multicolumn{2}{|c|}{$\begin{array}{l}<70 \text { years } \\
N=236\end{array}$} & \multicolumn{2}{|c|}{$\begin{array}{l}\geq 70 \text { years } \\
\mathrm{N}=1023\end{array}$} \\
\hline & $\mathbf{n}$ & $(\%)$ & $\mathbf{n}$ & $(\%)$ & $\mathbf{n}$ & $(\%)$ \\
\hline Capecitabine (xeloda) & 130 & 10.3 & 14 & 5.9 & 116 & 11.3 \\
\hline Carboplatin (paraplatin) & 143 & 11.4 & 30 & 12.7 & 113 & 11.0 \\
\hline Cyclophosphamide (cytoxan) & 360 & 28.6 & 97 & 41.1 & 263 & 25.7 \\
\hline Docetaxel (taxotere) & 290 & 23.0 & 73 & 30.9 & 217 & 21.2 \\
\hline Doxorubicin (adriamycin) & 256 & 20.3 & 79 & 33.5 & 177 & 17.3 \\
\hline Fluorouracil (adrucil) & 112 & 8.9 & 15 & 6.4 & 97 & 9.5 \\
\hline Gemcitabine (gemzar) & 87 & 6.9 & 18 & 7.6 & 69 & 6.7 \\
\hline Nab-paclitaxel (abraxane) & 68 & 5.4 & $* *$ & ** & $* *$ & ** \\
\hline Paclitaxel (taxol) & 295 & 23.4 & 60 & 25.4 & 235 & 23.0 \\
\hline Paclitaxel $($ taxol $)+$ other & 204 & 16.2 & 45 & 19.1 & 159 & 15.5 \\
\hline $\begin{aligned} \text { AC-T: } & {[\text { AC + paclitaxel (taxol) or }} \\
& \text { docetaxel (taxotere) }]\end{aligned}$ & 109 & 8.7 & 39 & 16.5 & 70 & 6.8 \\
\hline $\begin{array}{c}\text { AC: }[\text { doxorubicin (adriamycin) + } \\
\text { cyclophosphamide (cytoxan)] }\end{array}$ & 213 & 16.9 & 71 & 30.1 & 142 & 13.9 \\
\hline $\begin{array}{c}\text { TC: }[\text { docetaxel (taxotere) + } \\
\text { cyclophosphamide (cytoxan) or carboplatin }\end{array}$ & 143 & 11.4 & 38 & 16.1 & 105 & 10.3 \\
\hline Unknown chemotherapy & 73 & 5.8 & 12 & 5.1 & 61 & 6.0 \\
\hline
\end{tabular}

Note: Patients may receive more than one metastatic $1 \mathrm{~L}$ chemotherapy so percentages will add to more than $100 \%$; ${ }^{* *}$ Cells with counts of less than 11 are suppressed in compliance with the National Cancer Institute data use agreement for small cell sizes. 
Table 3. Time-varying Cox Model.

\begin{tabular}{|c|c|c|c|c|c|c|}
\hline \multirow[b]{2}{*}{ Chemotherapy } & \multicolumn{2}{|c|}{$\begin{array}{l}\text { Total metastatic } \\
\qquad N=2627\end{array}$} & \multicolumn{2}{|c|}{$\begin{array}{c}<70 \text { years }^{\mathrm{a}} \\
\mathrm{N}=359\end{array}$} & \multicolumn{2}{|c|}{$\begin{array}{l}\geq 70 \text { years }^{\mathrm{a}} \\
\mathrm{N}=2268\end{array}$} \\
\hline & HR & $95 \% \mathrm{CI}$ & HR & $95 \% \mathrm{CI}$ & HR & $95 \% \mathrm{CI}$ \\
\hline Treated & ref & & ref & & ref & \\
\hline Not treated & 1.095 & $1.00-1.20$ & 1.455 & $1.08-1.96$ & 1.166 & $1.06-1.29$ \\
\hline \multicolumn{7}{|l|}{ Age at diagnosis } \\
\hline $66-70$ & ref & ref & & & & \\
\hline $71-75$ & 1.095 & $0.95-1.26$ & & & & \\
\hline $76-80$ & 1.333 & $1.16-1.54$ & & & & \\
\hline$>80$ & 1.707 & $1.48-1.96$ & & & & \\
\hline \multicolumn{7}{|l|}{ Race/ethnicity } \\
\hline White & ref & & ref & & ref & \\
\hline Black & 0.863 & $0.76-0.98$ & 0.731 & $0.52-1.02$ & 0.851 & $0.74-0.98$ \\
\hline Others & 0.795 & $0.64-0.99$ & 0.760 & $0.36-1.60$ & 0.781 & $0.62-0.98$ \\
\hline \multicolumn{7}{|c|}{ Stage at initial diagnosis } \\
\hline Stage I & ref & & ref & & ref & \\
\hline Stage II & 1.196 & $1.05-1.36$ & 1.193 & $0.76-1.87$ & 1.208 & $1.06-1.38$ \\
\hline Stage III & 1.424 & $1.22-1.66$ & 1.825 & $1.10-3.02$ & 1.455 & $1.24-1.71$ \\
\hline Stage IV & 1.609 & $1.40-1.85$ & 2.170 & $1.38-3.42$ & 1.564 & $1.35-1.82$ \\
\hline \multicolumn{7}{|l|}{ PPI } \\
\hline No & ref & & ref & & ref & \\
\hline Yes & 1.180 & $1.03-1.35$ & 1.122 & $0.71-1.79$ & 1.215 & $1.05-1.41$ \\
\hline \multicolumn{7}{|c|}{ NCI comorbidity score } \\
\hline 0 & ref & & ref & & ref & \\
\hline 1 & 1.236 & $1.11-1.38$ & 0.773 & $0.55-1.09$ & 1.304 & $1.16-1.46$ \\
\hline 2 & 1.046 & $0.90-1.22$ & 1.268 & $0.74-2.17$ & 1.055 & $0.90-1.24$ \\
\hline$\geq 3$ & 1.424 & $1.21-1.67$ & 1.907 & $1.24-2.94$ & 1.339 & $1.12-1.60$ \\
\hline \multicolumn{7}{|l|}{ Radiotherapy } \\
\hline No & ref & & ref & & ref & \\
\hline Yes & 0.809 & $0.74-0.89$ & 0.972 & $0.73-1.29$ & 0.763 & $0.69-0.84$ \\
\hline \multicolumn{7}{|c|}{ Breast cancer surgery } \\
\hline No & ref & & ref & & ref & \\
\hline Yes & 0.618 & $0.55-0.70$ & 0.438 & $0.32-0.61$ & 0.663 & $0.58-0.76$ \\
\hline
\end{tabular}

Abbreviations: HR: Hazard Ratio; CI: confidence interval; NCI: National Cancer Institute; PPI: poor performance indicators. ${ }^{\mathrm{a} M o d e l}$ also includes marital status, education, income, geographic region, and year of diagnosis. 


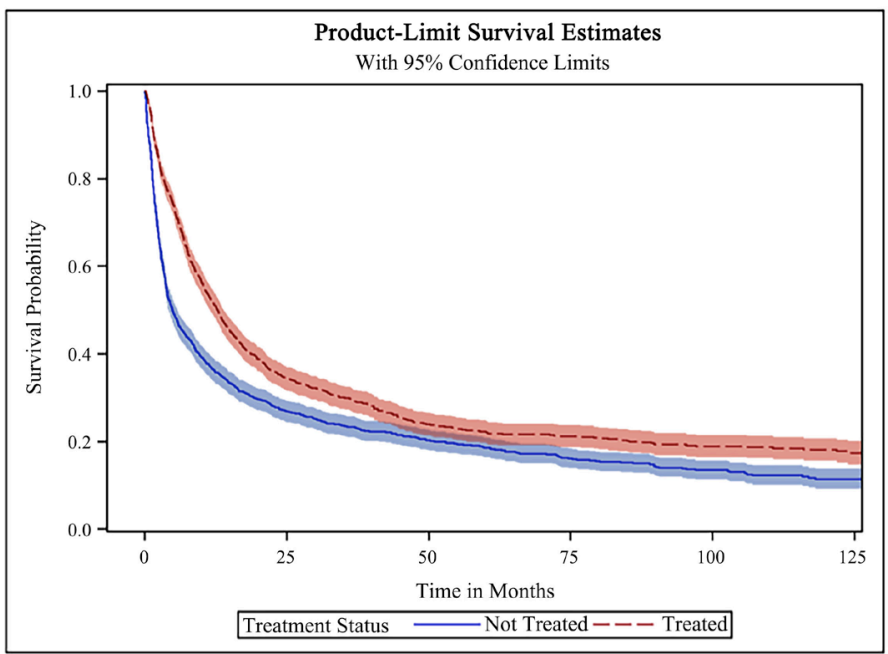

(a)

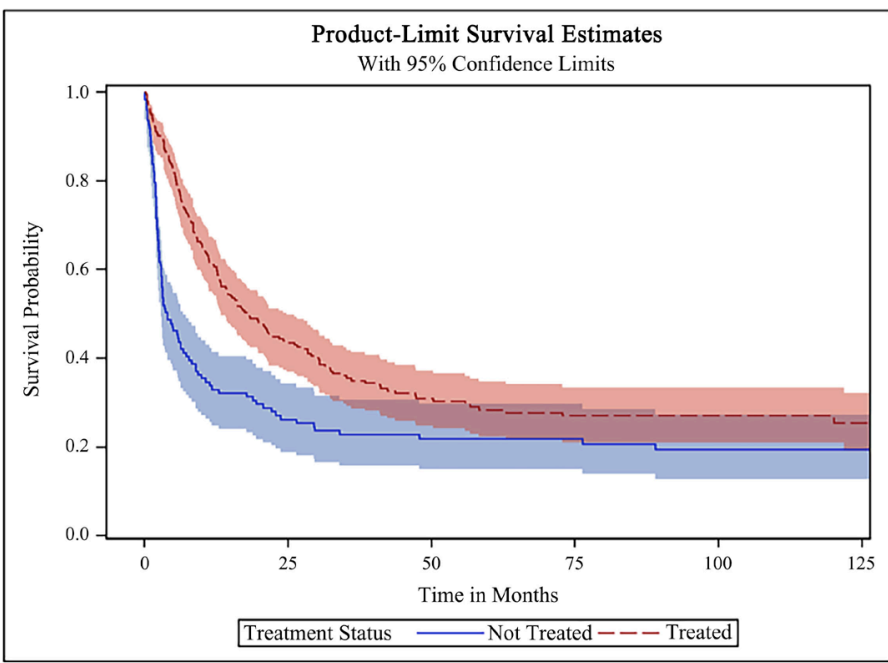

(b)

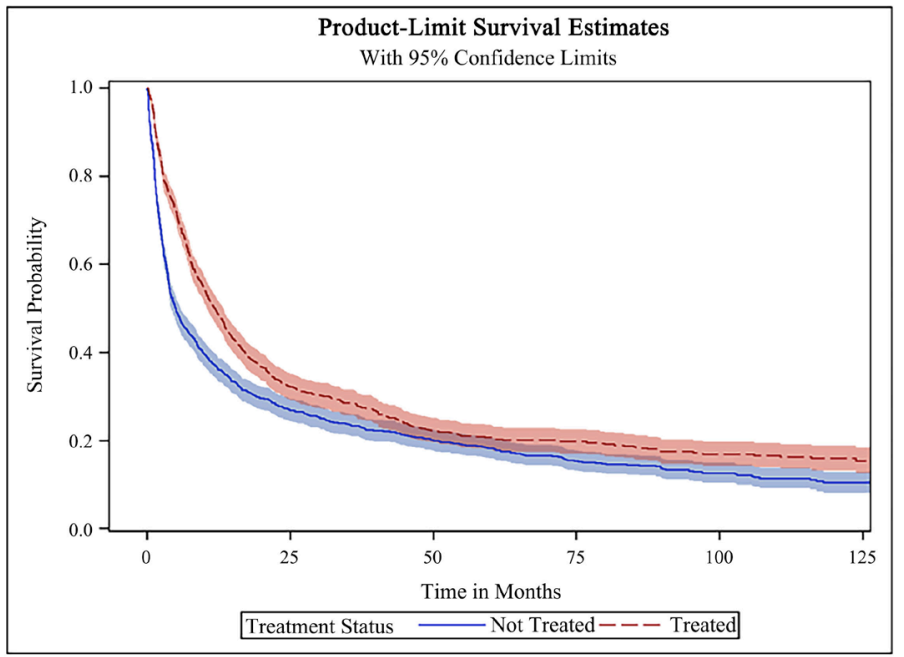

(c)

Figure 2. Unadjusted overall survival by treatment status. (a) All metastatic patients; (b) $<70$ year olds; (c) $\geq 70$ year olds. 


\subsection{De Novo vs. Non-De Novo Metastatic Treated Subgroup Analysis}

A subgroup analysis of the chemotherapy-treated cohort was performed to compare treatment patterns and outcomes between de novo patients $(\mathrm{N}=415)$ and non-de novo patients $(\mathrm{N}=844)$ who experienced distant metastasis or relapsed disease following an initial diagnosis with Stage I - III disease. Treatment for early breast cancer was defined as having received chemotherapy within 6 months after initial Stage I - III diagnosis. Patients with non-de novo metastatic disease were stratified into two groups: those who relapsed or progressed $\leq 12$ months after completing treatment for early breast cancer (fast relapse; $\mathrm{N}=250$ ) and those who relapsed or progressed $>12$ months after completing treatment for early breast cancer (slow relapse; $\mathrm{N}=594$ ). We found that patients who relapse slow were older and more likely to be initially diagnosed with Stage I disease compared to patients who relapse fast (Table 4). Overall, non de-novo metastatic patients were more likely to have prior radiotherapy and breast cancer

Table 4. Demographic and clinical characteristics by metastatic group.

\begin{tabular}{|c|c|c|c|c|}
\hline & \multicolumn{2}{|c|}{ Non-de novo metastatic } & \multirow{2}{*}{$\begin{array}{l}\text { De Novo metastatic } \\
\qquad \mathrm{N}=415\end{array}$} & \multirow[b]{2}{*}{$\mathrm{p}$ value } \\
\hline & $\begin{array}{l}\text { Fast relapse }{ }^{a} \\
N=250\end{array}$ & $\begin{array}{c}\text { Slow relapse }^{\mathrm{b}} \\
\mathrm{N}=594\end{array}$ & & \\
\hline Mean age $(95 \% \mathrm{CI})$ & $74.5(73.8-75.3)$ & $76.1(75.6-76.6)$ & $74.7(74.1-75.3)$ & $<0.0001$ \\
\hline \multicolumn{5}{|l|}{ Initial stage at diagnosis, $\%$} \\
\hline Stage I & 27 & 35 & - & $<0.0001$ \\
\hline Stage II & 42 & 43 & - & \\
\hline Stage III & 32 & 22 & - & \\
\hline Stage IV & - & - & 100 & \\
\hline \multicolumn{5}{|l|}{ Radiotherapy, \% } \\
\hline No & 28 & 25 & 50 & $<0.0001$ \\
\hline Yes & 72 & 75 & 50 & \\
\hline \multicolumn{5}{|l|}{ Surgery, \% } \\
\hline No & 8 & 6 & 51 & $<0.0001$ \\
\hline Yes & 92 & 94 & 49 & \\
\hline \multicolumn{5}{|l|}{ Chemotherapy, \% } \\
\hline Capecitabine & 5 & 11 & 13 & 0.0086 \\
\hline Carboplatin & 10 & 13 & 11 & 0.4948 \\
\hline Cyclophosphamide & 25 & 32 & 26 & 0.0397 \\
\hline Docetaxel & 23 & 21 & 26 & 0.2359 \\
\hline Doxorubicin & 16 & 23 & 19 & 0.0611 \\
\hline Paclitaxel & 18 & 24 & 27 & 0.0217 \\
\hline Median (IQR) duration of chemo, days & $58(30-128)$ & $116(72-164)$ & $102(50-149)$ & $<0.0001$ \\
\hline Median (95\% CI) OS, months & $12.1(9.4-16.0)$ & $19.9(16.4-24.6)$ & $7.8(6.7-8.9)$ & $<0.0001$ \\
\hline
\end{tabular}

${ }^{\mathrm{a}}$ Patients who relapsed or progressed $\leq 12$ months after completing treatment for early breast cancer; ${ }^{\mathrm{b}}$ Patients who relapsed or progressed $>12$ months after completing treatment for early breast cancer. 
surgery compared to patients with de novo Stage IV disease. Patients who relapse slow were more likely to receive cyclophosphamide and doxorubicin while docetaxel and paclitaxel were more commonly given to patients with de novo Stage IV disease. Median duration of chemotherapy was shortest for patients who relapse fast (58 days, IQR: 30 - 128), followed by patients with de novo Stage IV disease (102 days, IQR: 50 - 149), and longest for patients who relapse slow (116 days, IQR: 72 - 164; $\mathrm{p}<0.0001$ ). The median unadjusted OS was shortest for patients with de novo Stage IV disease (7.8 months, 95\% CI: 6.7 8.9), followed by patients who relapse fast (12.1 months, $95 \%$ CI: $9.4-16.0)$, and longest for patients who relapse slow (19.9 months, 95\% CI: 16.4 - 24.6).

\section{Discussion}

This large population-based study showed that elderly patients with metastatic TNBC were frequently untreated with over half not receiving chemotherapy. The study also demonstrated that patients who were 70 years and older were more likely to suffer from pre-existing comorbidities and had poorer performance compared to patients younger than 70 years old. These findings are consistent with other studies that have shown older age and co-morbidities are associated with less aggressive treatment [23] [24]. After adjusting for comorbidity burden, poor performance and other patient characteristics in the multivariate survival models, a $46 \%$ increased mortality risk for untreated patients compared to chemotherapy treated patients in the younger cohort of less than 70 year olds was seen. The benefit of chemotherapy persisted in the older cohort but was not as prominent as that seen in the younger cohort. Data from clinical trials suggest that older and younger women may experience similar survival benefits from chemotherapy, and age alone should not contraindicate the use of chemotherapy in older women who are in good health [25].

Chemotherapy has been the mainstay of systemic treatment for TNBC but there is no gold-standard regimen. Studies suggest that taxanes in particular have significant activity in the treatment of TNBC [26] [27] [28]. This study showed that taxane-based chemotherapy regimens, as well as cyclophosphamide- and anthracycline-based regimens were most commonly used in this time period. Further, patients in the younger cohort were more likely to receive chemotherapy across most regimen types with the exception of capecitabine, the use of which was more prevalent in the older cohort. One prior study showed that $35 \%$ of patients older than 65 years were offered chemotherapy and they were twice as likely as younger patients to reject chemotherapy out of fear for side effects [29]. Moving forward, it will be important to design clinical trials to address the therapeutic challenges that exist in this cohort of patients. While endocrine and HER2-targeted therapy are ineffective in this patient population, the use of immunotherapy has been gaining traction in TNBC, which has been shown to be more immunogenic compared to other breast cancer subtypes [30]. Early clinical experience with programmed cell death (PD-1) antibody pembrolizumab as well as the programmed cell death ligand (PD-L1) antibody atezoli- 
zumab show promising results in clinical trials [31] [32] [33].

This study also found that a history of radiotherapy and breast cancer surgery were associated with lower mortality risks. Age-related declines in major physiologic functions may impact a patient's ability to tolerate surgery, radiation, and cytotoxic chemotherapy [2]. However, studies that examined the predictors of receiving surgery and radiation found that increasing age was associated with substandard therapy independent of performance status or comorbidities, suggesting that physicians may be under-treating otherwise "healthy" elderly women [2].

In the subset analysis of treated patients, women with relapsed disease had higher unadjusted OS compared to women with de novo Stage IV disease. One prior study comprised of younger patients (median age $50-52$ years) of all breast cancer subtypes found the opposite effect [34]. However, after restricting their analysis to women with at least a 5-year disease free interval (time from primary non-metastatic diagnosis to first distant metastasis), relapsed patients exhibited a statistically significant longer OS compared to de novo Stage IV patients [34]. In the current study it was found that patients who relapse slow (disease-free interval of $>12$ months) had superior prognostic outcomes compared to patients who relapse fast (disease-free interval of $<12$ months). Disease-free interval or time to disease progression appears to be an important prognostic variable among women with relapsed disease [34].

\section{Strengths \& Limitations}

Clinical trial participants are often not representative of individuals in the real-world, and the SEER-Medicare database is therefore an invaluable tool for studying treatment patterns and long-term outcomes among elderly patients who have been historically underrepresented in clinical trials. The dataset includes a large sample size with diverse geographic representation of metastatic TNBC patients, and contains longitudinal claims data regardless of residence or service area from the time a person is eligible for Medicare until death. However, there are a few data limitations that should be addressed. The SEER registries do not collect follow-up information on disease progression after diagnosis, therefore metastasis or relapse could not be directly identified in the dataset. We used ICD-9 diagnosis codes indicative of secondary cancer in the Medicare claims to identify relapsed/progressed patients. However, using this method may result in incomplete or inaccurately coded information since these secondary cancer codes do not impact the amount of reimbursement to the provider. Several population-based studies have examined the completeness and accuracy of using ICD-9 diagnosis codes for secondary neoplasm and have reported considerable variability in the sensitivity, specificity and positive predictive value [35] [36]. Since the SEER registry did not have information on HER2 status prior to the year 2010, there was a large amount of missing data on HER2 status. Claims for HER2 targeted therapies were utilized as a proxy for HER2 status given that 
Medicare does not pay for targeted therapy without a positive HER2 test result. However, it's possible that patients who tested HER2 positive did not receive targeted therapy and could have been misclassified into the HER2 negative group. The SEER-Medicare database also does not include measures of performance status. Claims for oxygen and related respiratory supplies, wheelchair and supplies, home health agency services, and skilled nursing facility services were included as a surrogate for poor performance; however this may not adequately assess performance status for all patients in the study. Finally, information regarding treatment patterns and characteristics of patients enrolled in HMO or fee-for-service plans were not available since Medicare does not collect these data. Treatment patterns and survival outcomes may differ between these alternative health care plans and Medicare enrollees.

\title{
5. Conclusion
}

In this real-world analysis, roughly half of elderly TNBC patients did not receive chemotherapy following their metastatic diagnosis. Although the survival benefits of chemotherapy were stronger in the younger cohort, the benefits of treatment were maintained among $\geq 70$ year olds who were also less likely to receive chemotherapy. This may reflect under-treatment among elderly women with TNBC, potentially adversely affecting their prognosis. Elderly patients should be given guideline-based treatment in the absence of other reasons for withholding treatments. These results extend the findings from clinical trials conducted among younger women to an elderly breast cancer population, provide further insights into the natural history of the disease and remain unmet need in a real-world setting.

\section{Authors' Contributions}

\author{
Study Concepts: SS-H, CR. \\ Study Design: SS-H, PB, AS, PC, FM, CR. \\ Data Acquisition: SS-H, CR. \\ Quality Control of Data and Algorithms: SS-H, FM. \\ Data Analysis and Interpretation: SS-H, PB, AS, PC, FM, CR. \\ Statistical Analysis: SS-H, FM. \\ Manuscript Preparation: SS-H. \\ Manuscript Editing: SS-H, PB, AS, PC, FM, CR. \\ Manuscript Review: SS-H, PB, AS, PC, FM, CR.
}

\section{Acknowledgements}

This study was supported by Genentech, Inc. of South San Francisco, USA, a member of the Roche Group. This study used the linked SEER-Medicare database. We acknowledge the efforts of the Applied Research Program, NCI (Bethesda, MD), the Office of Information Services and the Office of Strategic Planning, Health Care Financing Administration (Baltimore, MD), Information Man- 
agement Services, Inc. (Silver Spring, MD), and the Surveillance, Epidemiology, and End Results (SEER) Program tumor registries in the creation of the SEER-Medicare database. The interpretation and reporting of these data are the sole responsibility of the authors.

\section{Conflicts of Interest}

$\mathrm{PB}, \mathrm{AS}, \mathrm{PC}$ and $\mathrm{CR}$ are employees of Genentech and shareholders of Roche. SS-H and FM work for Q.D. Research in a research and consulting capacity.

\section{References}

[1] Siegel, R.L., Miller, K.D. and Jemal, A. (2017) Cancer Statistics, 2017. CA: A Cancer Journal for Clinicians, 67, 7-30. https://doi.org/10.3322/caac.21387

[2] Tesarova, P. (2012) Breast Cancer in the Elderly-Should It Be Treated Differently? Reports of Practical Oncology and Radiotherapy, 18, 26-33. https://doi.org/10.1016/j.rpor.2012.05.005

[3] O’Shaughnessy, J. (2005) Extending Survival with Chemotherapy in Metastatic Breast Cancer. Oncologist, 10, 20-29. https://doi.org/10.1634/theoncologist.10-90003-20

[4] Foulkes, W.D., Smith, I.E. and Reis-Filho, J.S. (2010) Triple-Negative Breast Cancer. The New England Journal of Medicine, 363, 1938-1948.

https://doi.org/10.1056/NEJMra1001389

[5] Gonzalez-Angulo, A.M., Timms, K.M., Liu, S., et al. (2011) Incidence and Outcome of Brca Mutations in Unselected Patients with Triple Receptor-Negative Breast Cancer. Clinical Cancer Research, 17, 1082-1089. https://doi.org/10.1158/1078-0432.CCR-10-2560

[6] Carey, L.A., Perou, C.M., Livasy, C.A., et al. (2006) Race, Breast Cancer Subtypes, and Survival in the Carolina Breast Cancer Study. JAMA, 295, 2492-2502. https://doi.org/10.1001/jama.295.21.2492

[7] Dent, R., Trudeau, M., Pritchard, K.I., et al. (2007) Triple-Negative Breast Cancer: Clinical Features and Patterns of Recurrence. Clinical Cancer Research, 13, 4429-4434. https://doi.org/10.1158/1078-0432.CCR-06-3045

[8] National Comprehensive Cancer Network (2013) NCCN Clinical Practice Guidelines in Oncology. In: National Comprehensive Cancer Network, Ed., Breast Cancer, Version 2, NCCN, Fort Washington, PA.

[9] Hutchins, L.F., Unger, J.M., Crowley, J.J., et al. (1999) Underrepresentation of Patients 65 Years of Age or Older in Cancer-Treatment Trials. The New England Journal of Medicine, 341, 2061-2067. https://doi.org/10.1056/NEJM199912303412706

[10] Lewis, J.H., Kilgore, M.L., Goldman, D.P., et al. (2003) Participation of Patients 65 Years of Age or Older in Cancer Clinical Trials. Journal of Clinical Oncology, 21, 1383-1389. https://doi.org/10.1200/JCO.2003.08.010

[11] Mandelblatt, J.S., Hadley, J., Kerner, J.F., et al. (2000) Patterns of Breast Carcinoma Treatment in Older Women: Patient Preference and Clinical and Physical Influences. Cancer, 89, 561-573. https://doi.org/10.1002/1097-0142(20000801)89:3<561::AID-CNCR11>3.0.CO;2-A

[12] Harlan, L.C., Abrams, J., Warren, J.L., et al. (2002) Adjuvant Therapy for Breast Cancer: Practice Patterns of Community Physicians. Journal of Clinical Oncology, 
20, 1809-1817. https://doi.org/10.1200/JCO.2002.07.052

[13] Hebert-Croteau, N., Brisson, J., Latreille, J., et al. (1999) Time Trends in Systemic Adjuvant Treatment for Node-Negative Breast Cancer. Journal of Clinical Oncolo$g y, 17,1458-1464$. https://doi.org/10.1200/JCO.1999.17.5.1458

[14] Gennari, R., Curigliano, G., Rotmensz, N., et al. (2004) Breast Carcinoma in Elderly Women: Features of Disease Presentation, Choice of Local and Systemic Treatments Compared with Younger Postmenopasual Patients. Cancer, 101, 1302-1310. https://doi.org/10.1002/cncr.20535

[15] Warren, J.L., Klabunde, C.N., Schrag, D., et al. (2002) Overview of the Seer-Medicare Data: Content, Research Applications, and Generalizability to the United States Elderly Population. Medical Care, 40, IV-3-18. https://doi.org/10.1097/00005650-200208001-00002

[16] Stokes, M.E., Thompson, D., Montoya, E.L., et al. (2008) Ten-Year Survival and Cost Following Breast Cancer Recurrence: Estimates from Seer-Medicare Data. Value Health, 11, 213-220. https://doi.org/10.1111/j.1524-4733.2007.00226.x

[17] Lamont, E.B., Herndon, J.E., Weeks, J.C., et al. (2006) Measuring Disease-Free Survival and Cancer Relapse Using Medicare Claims from Calgb Breast Cancer Trial Participants (Companion to 9344). Journal of the National Cancer Institute, 98, 1335-1338. https://doi.org/10.1093/jnci/djj363

[18] Davidoff, A.J., Tang, M., Seal, B., et al. (2010) Chemotherapy and Survival Benefit in Elderly Patients with Advanced Non-Small-Cell Lung Cancer. Journal of Clinical Oncology, 28, 2191-2197. https://doi.org/10.1200/JCO.2009.25.4052

[19] Klabunde, C.N., Legler, J.M., Warren, J.L., et al. (2007) A Refined Comorbidity Measurement Algorithm for Claims-Based Studies of Breast, Prostate, Colorectal, and Lung Cancer Patients. Annals of Epidemiology, 17, 584-590. https://doi.org/10.1016/j.annepidem.2007.03.011

[20] Charlson, M.E., Pompei, P., Ales, K.L., et al. (1987) A New Method of Classifying Prognostic Comorbidity in Longitudinal Studies: Development and Validation. Journal of Chronic Diseases, 40, 373-383. https://doi.org/10.1016/0021-9681(87)90171-8

[21] Warren, J.L., Harlan, L.C., Fahey, A., et al. (2002) Utility of the Seer-Medicare Data to Identify Chemotherapy Use. Medical Care, 40, 55-61. https://doi.org/10.1097/00005650-200208001-00008

[22] Suissa, S. (2008) Immortal Time Bias in Pharmaco-Epidemiology. American Journal of Epidemiology, 167, 492-499. https://doi.org/10.1093/aje/kwm324

[23] Du, X. and Goodwin, J.S. (2001) Patterns of Use of Chemotherapy for Breast Cancer in Older Women: Findings from Medicare Claims Data. Journal of Clinical Oncology, 19, 1455-1461. https://doi.org/10.1200/JCO.2001.19.5.1455

[24] Newschaffer, C.J., Penberthy, L., Desch, C.E., et al. (1996) The Effect of Age and Comorbidity in the Treatment of Elderly Women with Nonmetastatic Breast Cancer. Archives of Internal Medicine, 156, 85-90. https://doi.org/10.1001/archinte.1996.00440010103014

[25] Muss, H.B., Woolf, S., Berry, D., et al. (2005) Adjuvant Chemotherapy in Older and Younger Women with Lymph Node-Positive Breast Cancer. JAMA, 293, 1073-1081. https://doi.org/10.1001/jama.293.9.1073

[26] Hayes, D.F., Thor, A.D., Dressler, L.G., et al. (2007) Her2 and Response to Paclitaxel in Node-Positive Breast Cancer. The New England Journal of Medicine, 357, 1496-1506. https://doi.org/10.1056/NEJMoa071167

[27] Hugh, J., Hanson, J., Cheang, M.C., et al. (2009) Breast Cancer Subtypes and Re- 
sponse to Docetaxel in Node-Positive Breast Cancer: Use of an Immunohistochemical Definition in the bcirg 001 Trial. Journal of Clinical Oncology, 27, 1168-1176. https://doi.org/10.1200/JCO.2008.18.1024

[28] Martin, M., Rodriguez-Lescure, A., Ruiz, A., et al. (2010) Molecular Predictors of Efficacy of Adjuvant Weekly Paclitaxel in Early Breast Cancer. Breast Cancer Research and Treatment, 123, 149-157. https://doi.org/10.1007/s10549-009-0663-Z

[29] Newcomb, P.A. and Carbone, P.P. (1993) Cancer Treatment and Age: Patient Perspectives. Journal of the National Cancer Institute, 85, 1580-1584. https://doi.org/10.1093/jnci/85.19.1580

[30] Iglesia, M.D., Vincent, B.G., Parker, J.S., et al. (2014) Prognostic b-Cell Signatures Using mrna-seq in Patients with Subtype-Specific Breast and Ovarian Cancer. Clinical Cancer Research, 20, 3818-3829.

https://doi.org/10.1158/1078-0432.CCR-13-3368

[31] Nanda, R., Chow, L.Q., Dees, E.C., et al. (2016) Pembrolizumab in Patients with Advanced Triple-Negative Breast Cancer: Phase ib Keynote-012 Study. Journal of Clinical Oncology, 34, 2460-2467. https://doi.org/10.1200/JCO.2015.64.8931

[32] Garcia-Teijido, P., Cabal, M.L., Fernandez, I.P., et al. (2016) Tumor-Infiltrating Lymphocytes in Triple Negative Breast Cancer: The Future of Immune Targeting. Clinical Medicine Insights: Oncology, 10, 31-39. https://doi.org/10.4137/CMO.S34540

[33] Wimberly, H., Brown, J.R., Schalper, K., et al. (2015) Pd-11 Expression Correlates with Tumor-Infiltrating Lymphocytes and Response to Neoadjuvant Chemotherapy in Breast Cancer. Cancer Immunology Research, 3, 326-332. https://doi.org/10.1158/2326-6066.CIR-14-0133

[34] Dawood, S., Broglio, K., Ensor, J., et al. (2010) Survival Differences among Women with De Novo Stage IV and Relapsed Breast Cancer. Annals of Oncology, 21, 2169-2174. https://doi.org/10.1093/annonc/mdq220

[35] Hassett, M.J., Ritzwoller, D.P., Taback, N., et al. (2014) Validating Billing/Encounter Codes as Indicators of Lung, Colorectal, Breast, and Prostate Cancer Recurrence Using 2 Large Contemporary Cohorts. Medical Care, 52, e65-e73. https://doi.org/10.1097/MLR.0b013e318277eb6f

[36] Nordstrom, B.L., Whyte, J.L., Stolar, M., et al. (2012) Identification of Metastatic Cancer in Claims Data. Pharmacoepidemiology and Drug Safety, 21, 21-28. https://doi.org/10.1002/pds.3247 